\title{
Retinitis Pigmentosa, Staphyloma and Myopia Syndrome-A Rare Entity
}

\author{
Shorya Vardhan Azad ${ }^{1}$, Amber Amar Bhayana ${ }^{1 *}$, Akshaya Balaji ${ }^{1}$ \\ ${ }^{1}$ Dr. RP Centre for Ophthalmic Sciences, AIIMS, New Delhi, India
}

*Corresponding Author: Amber Amar Bhayana, Senior Resident, Dr. Rajendra Prasad Centre for Ophthalmic Sciences, All India Institute of Medical Sciences, New Delhi, India; Email: amber.amar.bhayana@gmail.com

Received Date: 10-01-2021; Accepted Date: 08-02-2021; Published Date: 16-02-2021

Copyright $^{\Theta} 2021$ by Bhayana AA, et al. All rights reserved. This is an open access article distributed under the terms of the Creative Commons Attribution License, which permits unrestricted use, distribution, and reproduction in any medium, provided the original author and source are credited.

\begin{abstract}
Retinitis Pigmentosa (RP) is a common retinal dystrophy which causes progressive vision loss. Waxy disc pallor, arteriolar attenuation with mid-peripheral bony spicules form characteristic triad of the disease. Beginning as nyctalopia, the disease progresses to cause significant vision loss and visual field defect. Pathological myopia, staphyloma and cataract may be associated with the disorder as spectrum of MRCS syndrome which has a varied phenotypic presentation. We would like to report one such case.
\end{abstract}

\section{Keywords}

Retinitis Pigmentosa; Intraocular Pressures; Vitreoretinal Surgery; Myopia

\section{Introduction}

Retinitis Pigmentosa (RP) is one of the commonest retinal dystrophies characterized by triad of waxy disc pallor, arteriolar attenuation and bony spicules. However, clinical picture may vary due to its phenotypic variability. It may be syndromic or non-syndromic. Syndromic associations include Usher syndrome and Bardet Biedl syndrome being common whereas rare ones include Cohen, Jeune and Cockayne syndrome. Diagnosis is based on history of night blindness and, typical fundus findings give a clue to its diagnosis which is verified by 
electrophysiological tests. Currently no definitive treatment option is available, management thus is mainly supportive and includes photo-protection, vitaminotherapy and treatment of complications like cataract and macular edema [1,2]. Myopia is a well-known association of RP [3]. Posterior staphyloma is a diagnostic feature of pathological myopia occurring as a consequence of localized thinning and stretching of the ocular coats due to axial elongation. Here, we report a female with co-existing RP and posterior staphyloma [4].

\section{Case}

A 64 year old female presented with complaints of diminished vision since childhood. The patient underwent uneventful cataract surgery of both eyes 6 years back. On examination best corrected visual acuity in the Right Eye (RE) was 20/200 and in the Left Eye (LE) was 20/60. Intraocular Pressures (IOP) were within normal limits. Patient was pseudophakic bilaterally (lens in the bag and well centred) with rest of the anterior segment examination within normal limits. Fundus showed waxy disc pallor, arteriolar attenuation, bony spicules in mid periphery and posterior staphyloma bilaterally (Fig. 1). Staphyloma were confirmed on Swept Source Optical Coherence Tomography (SS-OCT) (Fig. 2). SS-OCT additionally revealed a focal area of Vitreo-Macular Traction (VMT) in LE (Fig. 2). Axial lengths on A-scan ultrasonography were $25.3 \mathrm{~mm}$ and $25.5 \mathrm{~mm}$ of the right and left eyes respectively. Electroretinograms showed reduced amplitudes bilaterally. A diagnosis of bilateral pathological myopia with RP was made. Patient was advised RE vitreoretinal surgery with VMT release after explained visual prognosis. However, patient refused for surgery and thus was prescribed low vision aids and has been kept on close follow up.
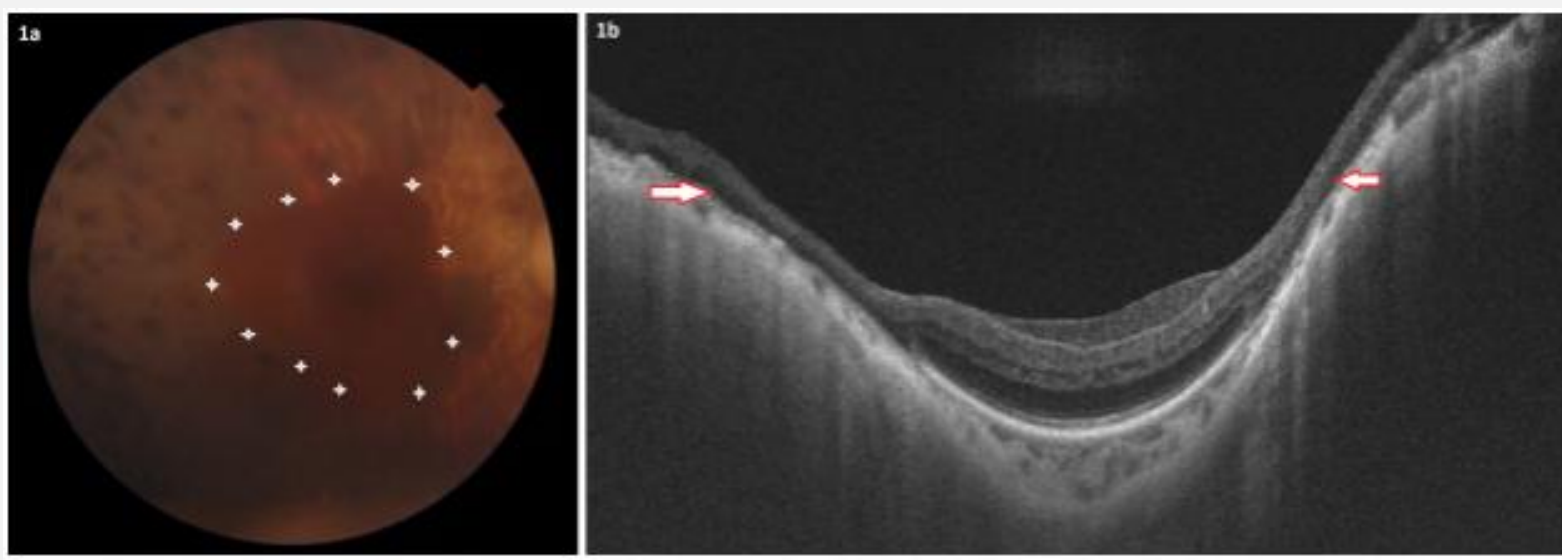

Figure 1: Right eye. Fundus photograph (a) shows bony spicules and margins of staphyloma (white stars). SSOCT (b) shows staphyloma's margin marked by red arrows. 

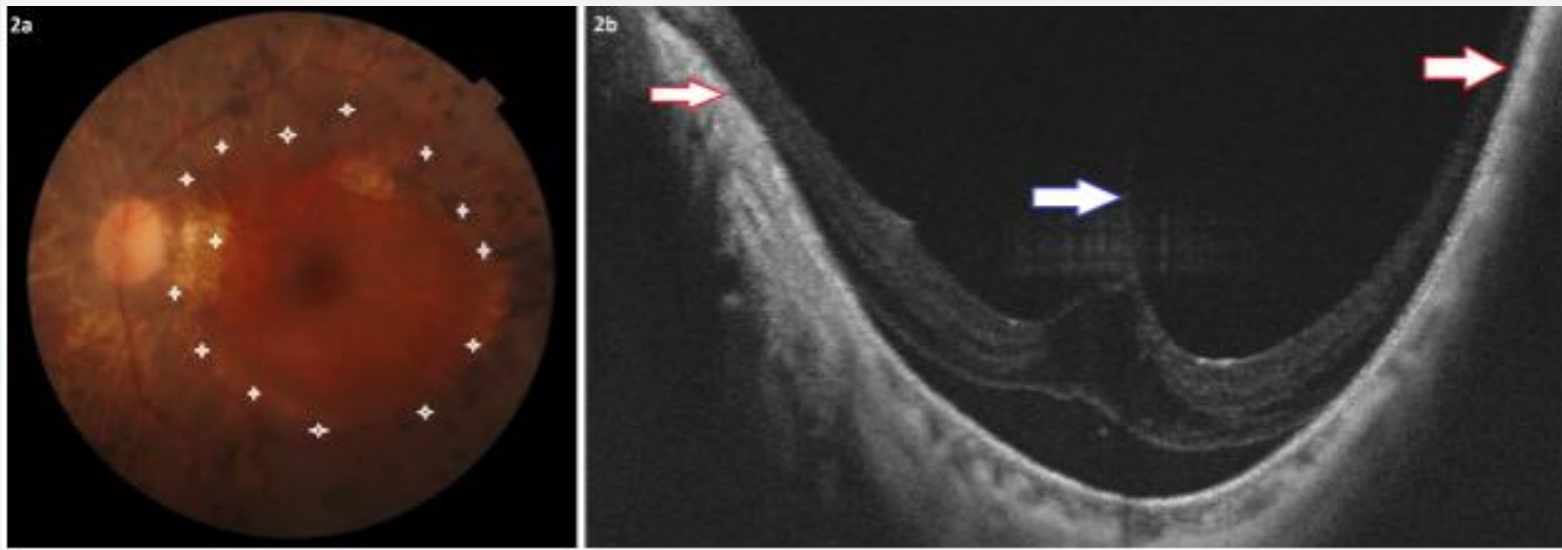

Figure 2: Left eye. Fundus photograph (a) shows disc pallor with bony spicules and margins of staphyloma (white stars). SSOCT (b) shows staphyloma's margin marked by red arrows and VMT shown by blue arrow.

\section{Discussion}

Pathological myopia is defined as refractive error greater than -6 diopters or axial length greater than $26.5 \mathrm{~mm}$. It is accompanied by degenerative changes in retina like posterior staphyloma, Foster-Fuch's spots, Lacquer cracks, peripapillary atrophy, chorioretinal atrophy etc. Posterior staphyloma is pathognomic of pathological myopia [4,5]. MRCS syndrome is combination of Myopia with RP (microcornea, retinal dystrophy, cataract and staphyloma) [6,7]. It is an autosomal recessive disorder. Vision loss presents in 20s-30s due to retinal dystrophy, cataract and posterior staphyloma. Retinal findings include retinal pigment deposition and retinal pigment epithelium atrophy. It is hypothesized to be caused by a mutation of NNO1 gene on chromosome 11 [7]. Our patient showed similar findings like cataract, posterior staphyloma and RP, supported by reduced ERG responses. MRCS syndrome doesn't have extinguished ERG responses as seen in our case. Also, microcornea was not present in our patient.

Staphyloma in our patient was of narrow macular type bilaterally. Choroidal thickness in our patient decreased from periphery upto the margin of staphyloma then again increased towards the centre of staphyloma. Co-existence of a typical RP with posterior staphyloma have been described in literature, albeit rarely. Ilhan, et al., described a case report with co-existing RP, moderate myopia and staphyloma, however, they did not document any OCT features. They used B-scan ultrasonography for diagnosis of staphylomas. Authors proposed it to be a subset of MRCS syndrome [8]. The patient had a refractive error of -6 dioptres in the right eye and 7 dioptres in the left eye. Cataract and microcornea were absent in their patient.

Similarly, Parmeggiani, et al., studied posterior staphylomas in 6 patients of RP, only 2 of which were high myopes and all had a narrow macular type of staphyloma [9]. Likewise, Xu, et al., studied 13 eyes of 11 patients having RP with axial length less than $26.5 \mathrm{~mm}$. All of them 
had narrow macular staphylomas, similar to our patient. Their OCT features and choroidal profile were also similar [10]. Matri, et al., studied 77 eyes of 39 retinitis pigmentosa patients and found posterior staphyloma in 17 eyes of 9 patients (all being non high myopes) [11]. 15 eyes had a narrow macular staphyloma and 2 eyes had a wide macular staphyloma. The reason for the development of posterior staphyloma and its chronology with respect to choroidal thinning in high myopes or RP patients remains elusive till now. Our case highlights the importance of recognizing incomplete expression of MRCS.

\section{Conclusion}

Co-existence of RP, cataract, myopia and posterior staphyloma may either be incomplete expression of MRCS syndrome, or it may fall within the spectrum of RP and myopia.

\section{References}

1. Gregory-Evans K, Weleber RG, Pennesi ME. Retinitis pigmentosa and allied disorders. In: Schachat AP, Ryan's Retina. Philadelphia: Elsevier. 2018:861.

2. Wang AL, Knight DK, Vu TT, Mehta MC. Retinitis pigmentosa: review of current treatment. Int Ophthalmol Clin. 2019;59(1):263-80.

3. Sieving PA, Fishman GA. Refractive errors of retinitis pigmentosa patients. Br J Ophthalmol. 1978;62:163-7.

4. Nagaoka N, Ohno-Matsui K, Saka N, Tokoro T, Mochizuki M. Clinical characteristics of patients with congenital high myopia. Jpn J Ophthalmol. 2011;55(1):7-10.

5. Kumar A, Chawla R, Kumawat D, Pillay G. Insight into high myopia and the macula. Indian J Ophthalmol. 2017;65(2):85-91.

6. Michaelides M, Urquhart J, Holder GE. Evidence of geneticheterogeneity in MRCS (microcornea, rodcone dystrophy,cataract, and posterior staphyloma) syndrome. Am J Ophthalmol. 2006;141:418-20.

7. Reddy MA, Francis PJ, Berry V. A clinical and molecular genetic study of a rare dominantly inherited syndrome (MRCS) comprising of microcornea, rod-cone dystrophy, cataract, andposterior staphyloma. Br $\mathrm{J}$ Ophthalmol. 2003;87:197-202.

8. Ilhan A, Yolcu U, Diner O, Uzun S, Gundogan FC. Coexistence of posterior staphyloma, retinitis pigmentosa and moderate myopia. J Coll Physicians Surg Pak. 2016;26:70-1.

9. Parmeggiani F, De Nadai K, Piovan A, Binotto A, Zamengo S, Chizzolini M. Optical coherence tomography imaging in the management of the Argus II retinal prosthesis system. Euro J Ophthalmol. 2017;27(1):16-21.

10. Xu X, Fang Y, Yokoi T, Shinohara K, Hirakata A, Iwata T, et al. Posterior staphylomas in eyes with retinitis pigmentosa without high myopia. Retina. 2019;39(7):1299-1304.

11. El Matri L, Falfoul Y, El Matri K, El Euch I, Ghali H, Habibi I, et al. Posterior staphylomas in non-highly myopic eyes with retinitis pigmentosa. Int Ophthalmol. 2020;40(9):2159-68. 